Proc. of the XI Int. Conf. - Ion Implantation and other Applications of Ions and Electrons, Kazimierz Dolny 2016

\title{
Ionisation Efficiency in Conical Hot Cavities
}

\author{
M. TUREK* \\ Institute of Physics, Maria Curie Skłodowska University in Lublin, \\ pl. M. Curie-Skłodowskiej 1, 20-031 Lublin, Poland
}

\begin{abstract}
Surface ionisation in a new type of hot cavity characterised by the shape of a truncated cone is considered in the paper. Influence of the hot cavity shape and its temperature on the ionisation efficiency of stable and long-lived nuclides is under investigation and the supremacy of elongated conical cavities is proven. The role of the extraction opening size is studied and its optimal diameter $(\approx 1 \mathrm{~mm})$ is found for the considered cavity shapes. It is shown that the extraction voltages of $1-2 \mathrm{kV}$ are large enough to obtain high efficiencies and saturation of the current-voltage curves is observed above these values. Changes of ionisation effciency with the ioniser temperature are investigated - increase of ion yield with $T$ is observed. It was proven that cold spots inside the ioniser cavity (near the extraction opening - in the considered case) can degrade ion source performance by several tens percent.
\end{abstract}

DOI: 10.12693/APhysPolA.132.259

PACS/topics: 07.77.Ka, 07.05.Tp, 34.35.+a

\section{Introduction}

Surface ionisation ion sources with hot cavities have attracted attention of engineers and scientists involved in different isotope separation on-line (ISOL) activities [1$3]$ for more than 40 years $[4,5]$, mainly due to such advantages as relatively high ionisation efficiency, purity of beam, low energy spread of extracted beam, and short time atoms stay in the ioniser (especially important in the case of short-lived isotopes). Their crucial part is a semi-opened ioniser heated to a high working temperature $(\approx 2000 \mathrm{~K})$ either by electron bombardment or ohmically $[6,7]$. It has usually the form of a tube but the spherical ones were also used $[8,9]$. In many solutions used in the ISOL facilities, the ioniser could be connected to a target by a transfer line $[8,10]$ or the ioniser itself is the irradiated target where new isotopes are produced and then released directly into the hot cavity [11].

In the previous papers a numerical model of hot cavity ion source was presented $[12,13]$. It is based on the assumption that multiple collisions of atoms with the hot ioniser walls could lead to high ion source ionisation efficiency. Electron impact ionisation was also proven to bring very important contribution for high ioniser temperatures and in the case of hard-to-ionise elements [14]. Several modifications were made to take into account radioactive decay of nuclides and delays due to the diffusion out of the target as well as effusion delay (related to sticking of particles to the ioniser walls) $[15,16]$. The model enabled studies of ion source efficiency as a function of many factors, like ioniser temperature, extraction voltage, timescales governing diffusion and effusion processes, as well as radioactive decay half-life. The ionisation calculations were performed for both tubular ioniser geometry and the spherical shapes [17, 18], which

*corresponding author; e-mail: mturek@kft.umcs.lublin.pl proved to be very effective, especially in the case of shortlived isotopes.

In this paper a new geometry of the ioniser is proposed. The hot cavity has a shape of a truncated cone and could be considered as transition between the elongated shapes of tubular ionisers and more compact shapes having advantage for short-lived nuclides. Moreover, one should keep in mind that conical cavity seems to be much easier to produce than a spherical one, as refractory metals are rather hard for machining. In the paper dependence of the ionisation efficiency on the hot cavity shape (mainly its elongation) is presented and discussed. The influence of the extraction opening diameter size on the ion yield is shown. The current-voltage curves obtained for different shapes of the cavity are compared. The dependence of the source efficiency on the ioniser temperature is also under investigation. It will be shown that maintaining possible the whole internal ioniser surface at high working temperature is crucial for obtaining high ionisation efficiency.

\section{Numerical model}

The numerical code follows the trajectories of neutral and charged particles inside the conically-shaped hot cavity of height $h$, and base radii $r$ and $R$. The flat extraction electrode on the $-V_{\text {ext }}$ potential is placed at the distance $d$ from the extraction opening of radius $r_{\text {ext }}$. Schematic view of the simulated system is shown in Fig. 1. The whole simulation area is covered by $800 \times 100 \times 100$ Cartesian numerical grid with the cell sizes $\Delta x=\Delta y=\Delta z=0.05 \mathrm{~mm}$. The electrostatic potential distribution is determined by discretization of the Laplace equation and solving it using the successive over-relaxation method, similarly to $[19,20]$. Boundary conditions are imposed by the shapes and voltages of electrodes. Particle trajectories are calculated by integration of classical equation of motion using the standard 4th order Runge-Kutta scheme. The electric field in the position of the particle of interest is calculated by linear 
interpolation of the field values at six nearest grid nodes. It is assumed that particles are emitted from the internal surface of the ioniser at random directions. Their initial velocities correspond to the ioniser temperature $T$. Collisions with other particles in the hot cavity are neglected within the considered model. The typical pressure in the cavity (for alkaline metals [21]) is below $10^{-4}$ mbar as the ioniser is usually made of refractory metals as well as due to the fact that very small amounts of substances are placed inside the cavity. Therefore, the typical mean path between collisions is larger than $1 \mathrm{~m}$, two or even three orders of magnitude larger than the average distance between the collisions with the ioniser surface.

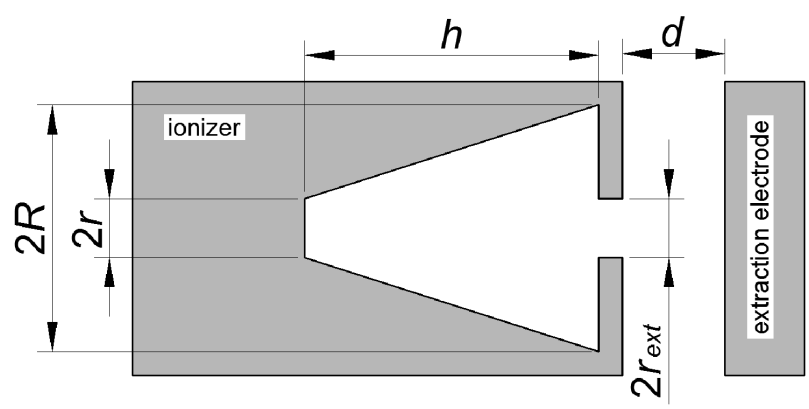

Fig. 1. Schematic view of the simulated system geometry.

A particle touching the ioniser wall could be ionised or neutralized with a probability given by the ionisation coefficient $\beta$, closely related to ionisation degree

$$
\beta=\alpha /(1+\alpha) \text {. }
$$

The $\alpha$ coefficient is defined as the ratio of the numbers of ionised and non-ionised atoms leaving the surface and could be calculated from the Saha-Langmuir formula

$$
\alpha=G \exp \left(-\left(V_{i}-\varphi_{e}\right) / k T,\right.
$$

where $V_{i}$ and $\varphi_{e}$ are the ionisation potential of an atom and the work function of the ioniser, respectively, and $G$ is a constant that depends on the ioniser surface and the ionised atom properties. The numerical code follows the trajectories of particles until they pass the extraction hole and counts the numbers of ions $\left(N_{+}\right)$and of neutrals $\left(N_{0}\right)$. Then the calculated ion source ionisation efficiency is defined as the ratio:

$$
\beta_{s}=\frac{N_{+}}{N_{+}+N_{0}} .
$$

This magnitude should not be mixed up with the ionisation probability $\beta$ defined for a single particle wall collision. Particles inside the cavity undergo many (tens, hundreds or even thousands, depending on the cavity geometry) collisions with the ioniser hot walls. This results in the increase of the total ion source efficiency defined by Eq. (3) much higher than the values predicted by the Saha-Langmuir law (2).

\section{Simulation results}

At first influence of the conical hot cavity shape on the total ionisation efficiency was studied. The cone height $h$ changed from $2.5 \mathrm{~mm}$ up to $30 \mathrm{~mm}$. The upper base radius $r$ was in the range from $0.2 \mathrm{~mm}$ up to $2 \mathrm{~mm}$ while the lower base radius $R$ was kept at $2.3 \mathrm{~mm}$. The flat extraction electrode $\left(V_{e x t}=-2 \mathrm{kV}\right)$ was placed at the distance $d=2 \mathrm{~mm}$ from the extraction opening. It was assumed that the particles are emitted with uniformly distributed probability from the lateral surface as well as from the upper base of the cavity cone. Calculations were done using 50000 particles of 150 a.m.u. mass. The simulation time-step was $10^{-8} \mathrm{~s}$. The ioniser temperature was set to $k T=0.3 \mathrm{eV}$. The results obtained for the $\beta$ coefficient changing over two decades (from 0.01 up to 1) are presented in Fig. 2. One can see that the total ionisation efficiency is much higher than that predicted by the Saha-Langmuir formula. This is especially well pronounced for the smaller ionisation coefficient - the amplification factor is $\approx 14$ for $\beta=0.01$ in the case of $h=2.5 \mathrm{~mm}$. One can see that the ionisation efficiency increases with the cone height reaching value $\beta_{s}=0.24$ for $\beta=0.01$ (amplification factor 24) in the case $h=30 \mathrm{~mm}$. Increase of the ionisation efficiency with $h$ for smaller $\beta$ could be explained by the fact that for the more elongated cavity shape hard-to-ionise particles can undergo more collisions than in the case of more compact shapes. This is confirmed by the data in Fig. 3. The mean number of particle-wall collisions increases by $\approx 20 \%$ for small $\beta$ as the cavity becomes more elongated.

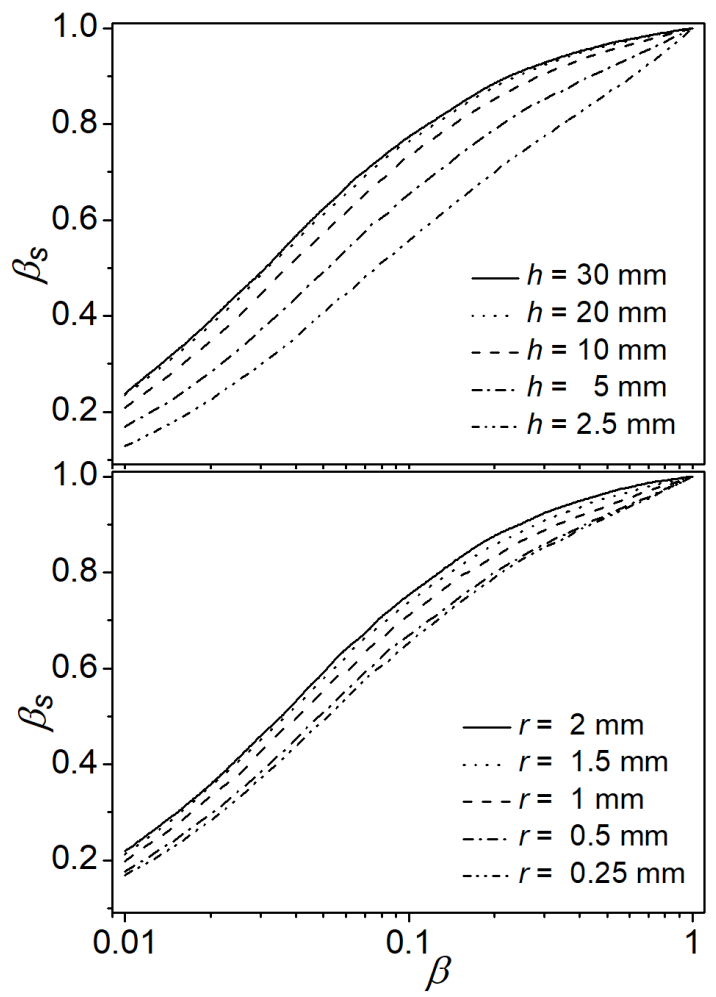

Fig. 2. Ionisation efficiency as a function of $\beta$ for different parameters describing the conical cavity: its height $h$ (a) and the upper base radius $r$ (b). 


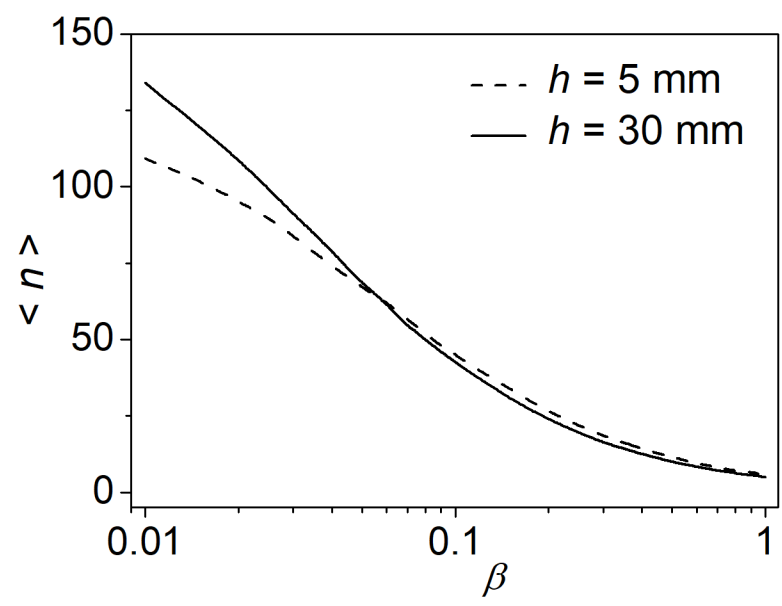

Fig. 3. The mean number of particle-wall collisions as a function of $\beta$ for two different cavity shapes.

The increase of ionisation efficiency with the upper base radius $r$ is also observed. One can see that the amplification factor for $\beta=0.01$ rises from $\approx 17(r=$ $0.2 \mathrm{~mm})$ up to $\approx 22(r=2 \mathrm{~mm})$. The increase could be explained in a similar way as in the previous case.

The changes of ionisation efficiency with the applied extraction voltage were also under investigation. Calculations were done for the two cavity shapes: the compact $(h=5 \mathrm{~mm})$ and the elongated one $(h=30 \mathrm{~mm})$. The other dimensions were $r=0.2$ and $R=2.3 \mathrm{~mm}$. The results for different values of $\beta$ coefficient are shown in Figure 4. In all cases one can see rapid increase of ion current with $V_{\text {ext }}$ rising up to approximately $1 \mathrm{kV}$. Saturation of current-voltage curves is observed when the extraction field is strong enough to capture and extract all ions almost immediately after they are produced. One should keep in mind that excessive (more than 1-2 keV for the considered $d$ distance) increase of the extraction voltage could lead to electrical breakdown. This is also pointless in the light of the presented data. The shape of the curves is similar to that obtained in the simulations for the spherical [17] and tubular [13] ionisers. It also resembles the experimental curves obtained for the tubular ioniser case [22]. It can be seen that the extracted currents in the whole $V_{\text {ext }}$ range are larger for the case of elongated cavity, which is in good accordance with the previously presented data.

The influence of the extraction aperture radius on the ionisation efficiency was also tested. Simulations were done for $r_{\text {ext }}$ changing in the range $0.2-1.2 \mathrm{~mm}$ and for the same as previously defined cavity geometries ( $h=5 \mathrm{~mm}$ and $h=30 \mathrm{~mm}$ ). All other simulation parameters were kept invariant. The results for different $\beta$ values are presented in Fig. 5. One can see that the optimal value is near $r_{\text {ext }}=0.5 \mathrm{~mm}$ in all considered cases. The presence of the maximum of $\beta\left(r_{\text {ext }}\right)$ is the result of two competing mechanisms. On the one hand, the smaller is the extraction hole, the more collisions with the

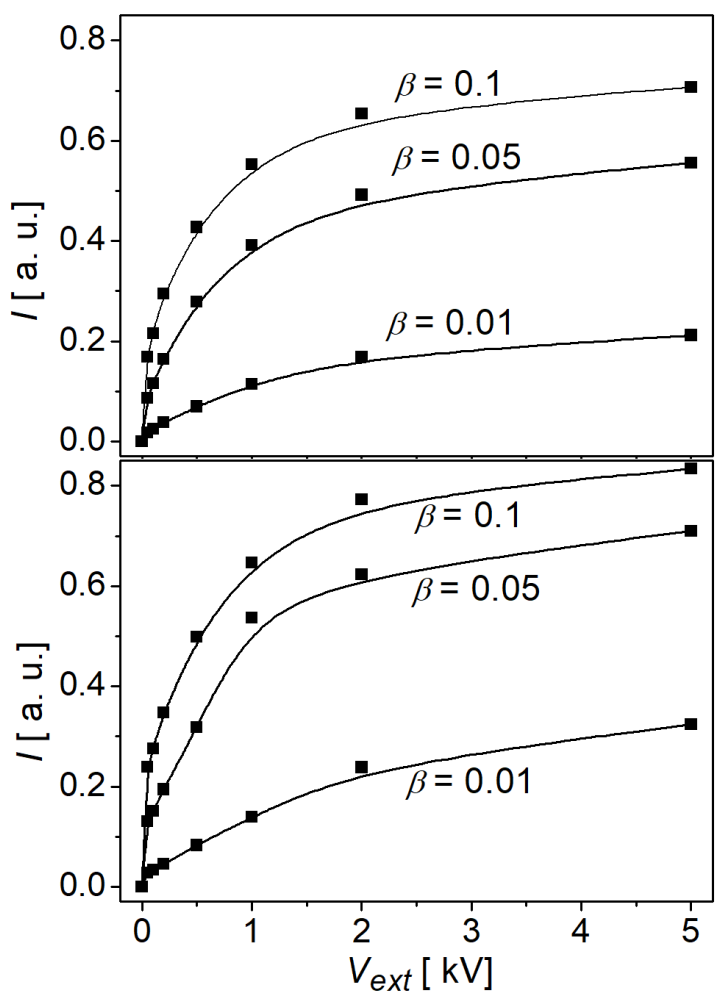

Fig. 4. Current-voltage curves obtained for the compact (a) and the elongated (b) cavity shapes.

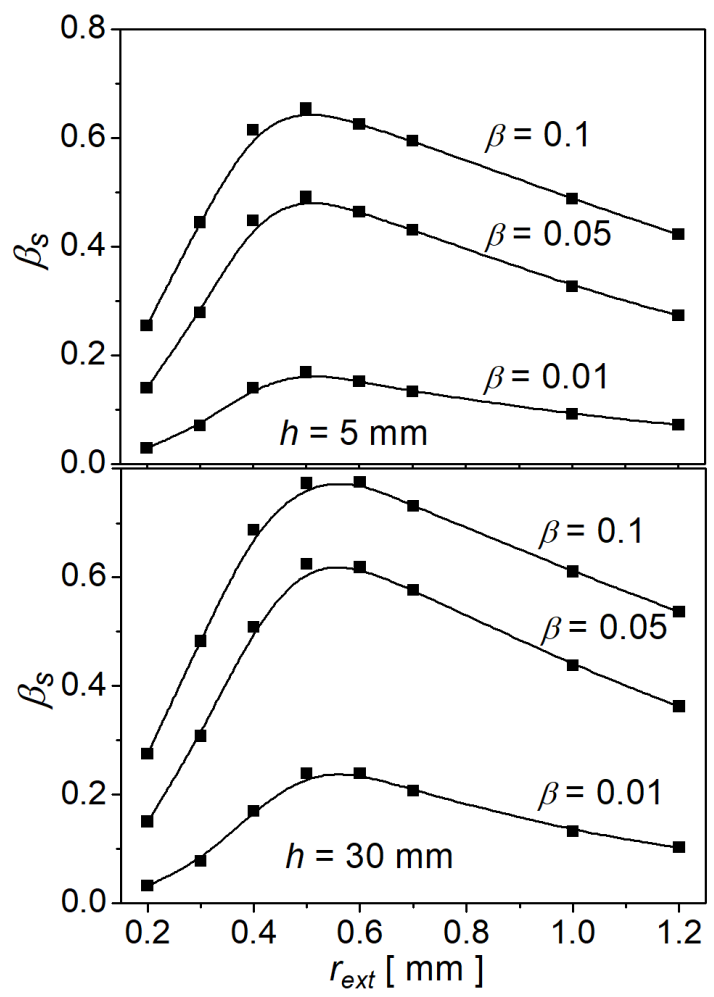

Fig. 5. Ionisation efficiency as a function of extraction opening size for the compact (a) and the elongated (b) cavity shapes. 
ioniser walls a particle undergoes (on average), which leads to the increased ionisation efficiency. In other words, the collision with the flat end-cap surface acts directs the particle backwards into the cavity. On the other hand, the larger is the extraction opening diameter, the more effective is the ion extraction, as the extraction field penetrates the cavity at larger depth. As a result, larger part of the cavity works in "each ion" regime [12] defined as a mode when each ion is removed from the ioniser immediately after its creation. One can note that the optimum in the case of $h=30 \mathrm{~mm}$ is slightly shifted towards larger $r_{\text {ext }}$, meaning that the balance larger "each ion" volume is required for more elongated cavity.

The numerical code was also used to predict dependences of ionisation efficiency on the ioniser temperature $k T$. Calculations were done for the cone height $h$ changing in the range from $2.5 \mathrm{~mm}$ up to $30 \mathrm{~mm}$, while $r=0.2 \mathrm{~mm}$. All other simulation parameters were kept the same as in the previously presented cases. Ioniser temperature was changed in the range from 0.2 up to $0.32 \mathrm{eV}$. One should keep in mind that not only initial velocity of the desorbing particle changes with the ioniser temperature, but also the ionisation probalility, as predicted by formulae (1) and (2). The ionisation degree for $k T=0.2 \mathrm{eV}$ was arbitrarily set to 0.01 , while $V_{i}-\varphi_{e}$ difference was chosen as $0.5 \mathrm{eV}$ which could be considered as a typical value. The simulation results are shown in Fig. 6. The relative increase of ionisation efficiency is presented. One can see the increase of $\beta_{s}$ with temperature which was also observed in experiments [23]. The relative increase is higher for the compact cavity shapes. It reaches $75 \%$ for the highest considered temperature in the case of $h=2.5 \mathrm{~mm}$. The corresponding increase for the elongated cavity is $\approx 50 \%$.

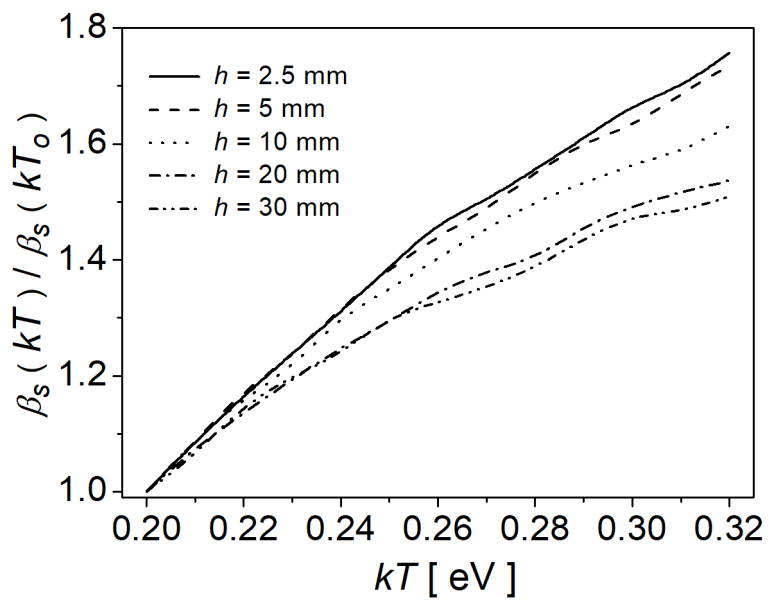

Fig. 6. Relative increase of ionisation efficiency with the ioniser temperature.

The calculations presented above were done under assumption that the flat end-cap is significantly colder than the rest of the ioniser and re-emits neutral atoms only. In other words it is assumed that the ionisation degree

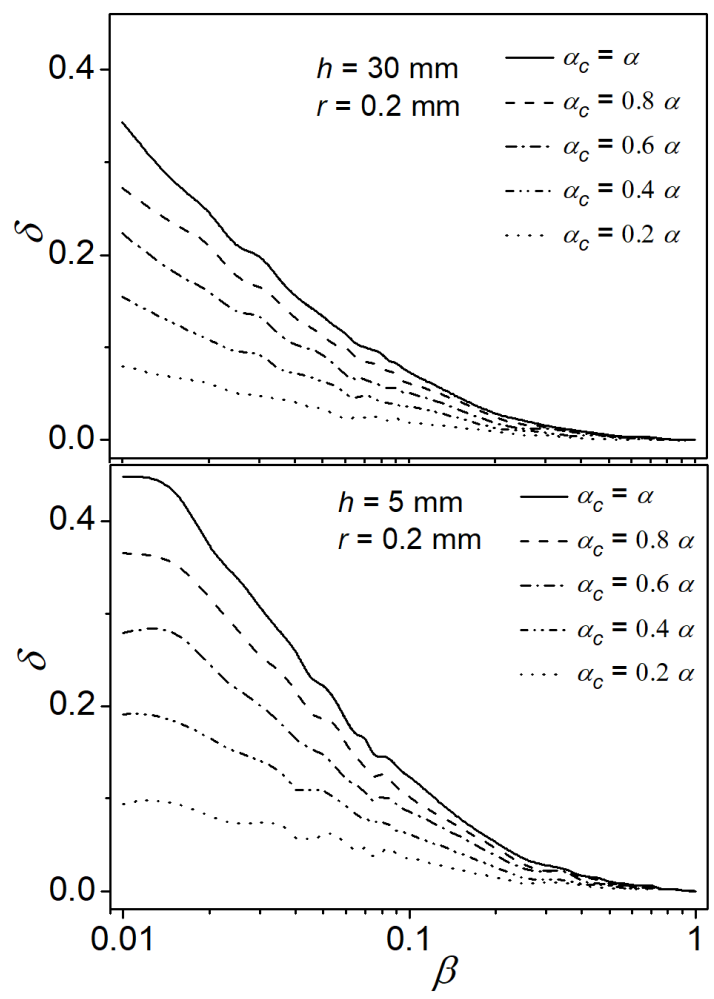

Fig. 7. Relative change of the ionisation efficiency with the temperature of the flat end-cap near the extraction opening. Results for the elongated (a) and the compact (b) geometry.

for that surface is $\alpha_{c}=0$. Uniform heating of the ioniser (especially near the extraction opening) is a crucial problem in hot cavity ion source design and engineering [6]. Colder spots can lead to efficiency degradation, as ionisation probability usually decreases in such places. This is confirmed by the simulation results for the two different shapes of conical cavity shown in Fig. 7. The relative increase of ionisation efficiency is defined as:

$$
\delta=\frac{\beta_{s}\left(\alpha_{c}\right)-\beta_{s}\left(\alpha_{c}=0\right)}{\beta_{s}\left(\alpha_{c}=0\right)} .
$$

It can be seen that the harder to ionise the substance is the more important is maintaining sufficiently high temperature also in the region of the extraction aperture. Keeping the temperature of the end-cap high enough, i.e. resulting in $\alpha_{c} \approx \alpha$ enables the increase of the ionisation efficiency by $\approx 30 \%$ (compared to the cold end-cap case) for the elongated cavity or even by $50 \%$ for the compact one.

\section{Conclusions}

A new geometry of hot cavity ioniser was proposed in the paper. The conical shape of the cavity could be considered as an intermediate form between the tubular and spherical ones. Non-extensive parametric studies of the ionisation efficiency as a function of the cavity shape were 
performed for the stable and long-lived isotopes. The advantage of elongated cavity shapes over the compact ones was proved, the twofold increase of ionisation efficiency is especially important in the case of substances characterised by small values of the ionisation probability coefficient $\beta$. The analysis of current-voltage curves obtained for different cavity shapes suggest that extraction voltages of $1-2 \mathrm{keV}$ are sufficient to remove ions efficiently. The changes of the ionisation efficiency with the size of the extraction opening were also studied - it was found that $r_{\text {ext }} \approx 0.5 \mathrm{~mm}$ is optimal in all considered cases. The influence of ioniser temperature on the ion source efficiency was also tested. The importance of maintaining high temperature on the whole ioniser surface was proved, inasmuch the presence of cold spots can degrade ion source performance by several tens of percent.

\section{References}

[1] V.N. Panteleev, Rev. Sci. Instrum. 75, 1602 (2004).

[2] G.D. Alton, Y. Liu, D.W. Stracener, Rev. Sci. Instrum. 77, 03A711 (2006).

[3] U. Köster, O. Arndt, E. Bouquerel, V.N. Fedoseyev, H. Franberg, A. Joinet, C. Jost, I.S.K. Kerkines, R. Kirchner and the TARGISOL Collaboration, Nucl. Instrum. Methods Phys. Res. B 266, 4229 (2008).

[4] G.J. Beyer, E. Herrmann, A. Piotrowski, V.I. Raiko, H. Tyroff, Nucl. Instrum. Methods 96, 437 , (1971).

[5] P.G. Johnson, A. Bolson, C.M. Henderson, Nucl. Instrum. Methods 83, 106 (1973).

[6] L. Zhai, H. Deng, G. Wei, Z. Li, C. Wang, X. Li, G. Zhou, Y. Su, Z. Zhang, Int. J. Mass Spectrom. 305, 45 (2011).
[7] C. Eléon, P. Jardin, G. Gaubert, M. Saintlaurent, J. Alcantaranunez, R. Alvesconde, Nucl. Instrum. Methods Phys. Res. B 266, 4362 (2008).

[8] G.D. Alton, Y. Liu, H. Zaim, S.N. Murray, Nucl. Instrum. Methods Phys. Res. B 211, 425 (2003).

[9] P.A. Hausladen, D.C. Weisser, N.R. Lobanov, L.K. Fifield, H.J. Wallace, Nucl. Instrum. Methods Phys. Res. B 190, 402 (2002).

[10] G.D. Alton, Y. Zhang, Nucl. Instrum. Methods Phys. Res. A 539, 540 (2005).

[11] V.G. Kalinnikov, K.Ya. Gromov, M. Janicki, Yu.V. Yushkevich, A.W. Potempa, V.G. Egorov, V.A. Bystrov, N.Yu. Kotovsky, S.V. Evtisov, Nucl. Instrum. Methods Phys. Res. B 70, 62 (1992).

[12] M. Turek, K. Pyszniak, A. Drozdziel, J. Sielanko, Vacuum 82, 1103 (2008).

[13] M. Turek, A. Drozdziel, K. Pyszniak, D. Maczka, B. Slowinski, Rev. Sci. Instrum. 83, 023303 (2012).

[14] M. Turek, K. Pyszniak, A. Droździel, Vacuum 83, S260 (2009).

[15] M. Turek, Acta Phys. Pol. A 123, 847 (2013).

[16] M. Turek, Vacuum 104, 1 (2014).

[17] M. Turek, Acta Phys. Pol. A 120, 188 (2011).

[18] M. Turek, Acta Phys. Pol. A 128, 935 (2015).

[19] A. Pyszniak, A. Droździel, M. Turek, A. Latuszyński, D. Maczka, J. Sielanko, Yu.A. Vaganov, Yu.V. Yushkevich, Instrum. Exp. Techn. 50, 552 (2007).

[20] M. Turek, J. Sielanko, Vacuum 83, S256 (2009).

[21] P. Van Duppen, Lect. Notes Phys. 700, 37 (2006).

[22] A. Latuszyński, D. Mączka, Vacuum 51, 109 (1998).

[23] A. Latuszyński, V.I. Raiko, Nucl. Instrum. Methods 125, 61 (1975). 